\title{
Perception and Experience of In-Sequence and Out-of- Sequence Engineering Students in a General Chemistry Laboratory
}

\author{
Courtney Spillman, Lorelie Imperial, \& Kent Crippen \\ University of Florida
}

Faculty mentor: Kent Crippen, College of Education

\begin{abstract}
ChANgE Chem (NSF-1625378) utilizes Cognitive Apprenticeship as a theoretical framework for integrating engineering practices into a freshman chemistry laboratory course for engineering majors with the goal of better supporting all students to degree completion. The activities are structured as three-week Design Challenges (DCs) where students use chemistry knowledge to solve authentic engineering problems. This study explores the experiences of students taking the course in-sequence (i.e. fall of freshman year) versus those taking it out-of-sequence (i.e. spring), where out-of-sequence students have been identified as at higher academic risk. Data was collected through audio and video recordings and postlaboratory surveys. Video recordings were coded using a protocol to identify type and frequency of issues and questions asked. The post-laboratory surveys obtained information concerning students' perception of task difficulty and their feelings of being like an engineer. The data demonstrated that while out-ofsequence students ask more questions and experience more issues, they did feel like successful engineers and did not find the tasks too difficult. Therefore, additional curriculum supports as well as assistance from a Teaching Assistant are needed in order to positively influence the persistence of out-of-sequence students in spite of the challenges they may face.

Keywords: chemistry laboratory, engineering, in-sequence, out-of-sequence students, situated learning
\end{abstract}

\section{Introduction}

At the University of Florida, General Chemistry 1 Laboratory is one of the common critical tracking courses for a variety of majors, especially engineering. Therefore, most STEM students will take the course in their first academic year, and success is considered essential for continuing to be on pace for graduation. Students who take the course in the Fall semester of their freshman year are considered in-sequence, whereas those who take the courses in the Spring semester or beyond are considered off-track or out-of-sequence. This study evaluates the capacity for supporting student persistence regardless of sequence, by comparing their experience in each situation. 
Research shows that approximately $40 \%$ of first year engineering students change their major by the end of their first year at university (Ohland, Sheppard, Lichtenstein, Eris, Chachra, \& Layton, 2008). The Cognitive Apprenticeship for Engineers in Chemistry Laboratory project (ChANgE Chem: NSF-1625378) aims to reduce these numbers by creating a unique engineering section of Gen Chem Lab 1 and 2 where the experience is designed so as to help students see themselves as apprentice engineers. Traditionally, general chemistry is viewed as one of the courses that discourages students to continue pursuing an engineering major as the course is viewed as difficult and typically not aligned to their goals. By contextualizing the laboratory within real-world engineering problems as Design Challenges (DC), students are provided with the needed support to help them identify as engineers early in their academic career. If successful, this intervention should be especially meaningful for the academically at-risk out-ofsequence students. By supporting their goal to become successful engineers, even if they are a semester behind in course tracking, the contextualized laboratory is expected to reduce the number of these students who elect to change their major.

A previous study by the ChANgE Chem team revealed that out-of-sequence students in this laboratory course were at higher academic risk, having lower levels of motivation across the semester (Payne, et al. 2019). Additionally, the institutional bias against out-of-sequence students due to their status as being off-track for graduation may negatively impact these students even though they may be taking the course out-of-sequence for a variety of reasons unrelated to academics. Therefore, this study took a closer look into a subset of participants to compare the experience and perceptions of the DCs between the in- and out-of-sequence students. One of the pillars of ChANgE Chem is supporting underrepresented and at-risk students in their academic endeavors, so identifying how out-of-sequence students experience the DC could offer insight into needed revisions as well as how the Teaching Assistants (TAs) and professors can better ensure that all students have the tools to succeed and feel that they are participating in relevant engineering experiments.

\section{Research Questions}

This study aims to gain insights regarding the two research questions (RQs):

1. How does student perception of a DC experience, namely feelings of success and perceived difficulty of task, differ between in- and out-of-sequence students? 
2. How does student experience in a DC, such as number of questions asked and number of issues, differ between in-sequence and out-of-sequence students?

\section{Theoretical Framework: Cognitive Apprenticeship}

ChANgE Chem supports the re-design of the traditional freshman general chemistry laboratory activities into a structure called a DC that incorporates three important elements of authentic engineering practice, namely, engineering design process, real-world context and collaboration while ensuring learning of the chemistry content. We chose to focus on the laboratory component of the general chemistry course because laboratory activities have long been seen as important components of chemistry curricula (Hofstein \& Lunetta, 2004). The laboratory environment offers unique opportunities for students to form links between theoretical phenomena and experimental modeling of chemistry concepts. For engineering majors, situating these activities in authentic practice strengthens the connection between the domain knowledge of chemistry and its application in everyday work activities (Popper, 1999).

Central to the development of a DC is the use of Cognitive Apprenticeship (Johri \& Olds, 2011) as the theoretical framework. Use of this framework allowed the meaningful contextualization of the chemistry content within the engineering context. DCs were developed integrating the elements of authentic engineering practices previously mentioned. The DCs were designed with the intent of retaining engineering students by creating engaging and relevant integrated engineering and chemistry activities in a collaborative laboratory setting. Contextually relevant learning is theorized to maintain student interest and encourage students to persist towards completing their engineering degree (Krapp \& Prenzel, 2011).

\section{Real-World Context: Design Challenge 2: Making Solar Energy Economical}

DCs are three-week collaborative laboratory activities divided into Design, Conduct, and Analyze phases. This study focuses on the second DC, DC2: Make Solar Energy Economical, one of the NAE Grand Challenges for Engineering. (National Academy of Engineering, n.d.). For DC2, the students were tasked to use the concept of specific heat capacity to build a concentrating solar power (CSP). Students tested a variety of metals and compounds to create a recommendation regarding the best heat storage materials while staying within certain cost and tower height constraints. During the Design phase, students obtain background information on the engineering challenge and perform preliminary qualitative tests. They use the results of these tests and gather more quantitative evidence during the Conduct phase. For DC2, students measured specific heat 
capacities using a solar thermal collector they constructed and optimized during the Design phase. Data analysis, test refinement, and creation of the final deliverable in the form of a Technical Memo is completed during the Analyze phase. At the end of each phase, students reason collaboratively and construct an argument using a Claim-Evidence-Reasoning (CER) format. The CER documentation ensures that students use their data and chemistry knowledge to provide evidence to make a recommendation for the best material to build the CSP.

\section{Methodology}

We used a comparative case study by randomly selecting two teams (five students) from each semester to include in the analysis of this paper.

\section{Participants}

The participants were freshman engineering students who self-selected to enroll in the special General Chemistry 1 Laboratory during Fall 2017 and Spring 2018 semesters. They consented to participate in the study (UF-IRB201600944) at the beginning of the semester. Of the ten participants, there were eight engineering majors represented (environmental, electrical, aerospace, civil, industrial, computer science/engineering, biomedical, and chemical). Six of the participants identified as male and four as female. Five of the participants identified their ethnicity as white, one as Hispanic and white, one as black or African American, one as Asian and white, and one as Hispanic.

\section{Data Collection and Analysis}

\section{RQ1}

Data concerning students' perception of the DC was collected through an exit ticket, which is a ten-item survey that the participants completed at the end of each laboratory phase. Participants responded by indicating their degree of agreement on 5-point Likert-type scale. We collected and analyzed the responses to two questions pertaining to level of difficulty and feeling like a practicing engineer. We calculated the average response for the two survey questions and compared the results between groups across the three DC phases (Tables 1-3).

\section{RQ2}

A Swivl robot and iPad was used to obtain video and audio recordings of participants as they completed laboratory tasks. Audio recorders were placed in multiple areas to capture small group conversations. In order to track common laboratory issues and compare progress between groups, audio and video footage were reviewed and coded by research team members. 
Completing this analysis involved a video coding protocol (Appendix A) which lists and defines the types of issues and questions raised as participants worked with the materials. As the videos were reviewed, any questions asked by the students were coded as Student questions and help or questions prompted by the teaching assistant (TA) were coded as TA questions. Any issue regarding scientific, mathematical, or engineering concepts as well as any issue regarding the prescribed sets of steps or protocol was coded as Issue. We then calculated the frequency of both issues and questions and compared the results between groups across the three DC phases (Tables 1-3).

limitations for video coding. Due to a lack of useable audio for one of the Spring 2018 Design videos, data for the out-of-sequence group only consists of questions and issues from two participants. Recording issues for one of the Fall 2017 Conduct videos resulted in the video being cut off at around one hour, therefore data for each Conduct video was also collected up to that mark for equal comparisons.

\section{Results}

Design Phase. Table 1 shows the results from the post-phase survey and video coding.

Table 1. Post Survey and Video Coding Results for Design Phase

\begin{tabular}{lcc}
\hline & $\begin{array}{c}\text { In-Sequence } \\
\mathrm{n}=5\end{array}$ & $\begin{array}{c}\text { Out-of-Sequence } \\
\mathrm{n}=2\end{array}$ \\
\hline Level of Difficulty & 14 & 6 \\
$\quad$ Total & 2.8 & 2.8 \\
Average & 0.84 & 1.41 \\
SD & & \\
Feel Like Engineer & 21 & 7 \\
Total & 4.2 & 4.4 \\
Average & 0.84 & 0.71 \\
SD & & \\
Student Questions & 24 & 24 \\
Total & 4.8 & 12 \\
Average & 4.38 & 0 \\
SD & & \\
TA Questions & 6 & 14 \\
Total & 1.2 & 7 \\
Average & 1.64 & 0 \\
SD & & \\
Issues & 37 & 74 \\
Total & 7.4 & 0 \\
Average & 0.55 & 7 \\
SD & & \\
\hline
\end{tabular}

Note. Spring 2018 P5 Video unusable. Data from only two participants was obtained. 
The average self-reported perceptions regarding the DC Design phase in terms of level of difficulty and feelings of being like an engineer were similar for both the in- and out-of-sequence groups.

The out-of-sequence students, on average, asked noticeably more questions than the insequence students. These results demonstrate that the out-of-sequence students needed more assistance in completing the tasks for the Design phase compared to the in-sequence students. Similarly, there were more help requests and questions prompted by the TA towards the out-ofsequence group.

Despite the differences in the frequency of questions, the average issues per participant were similar between the two groups. This suggests that an increase in questions during the Design phase does not necessarily correlate to an increase in issues or lack of conceptual understanding. Examples of the issues common to both in- and out-of-sequence groups in this phase were trouble setting up the calorimeter correctly and performing heat capacity calculations. The outof-sequence participants had an additional issue remembering to write down their initial temperature reading, which further complicated the heat capacity calculations.

Conduct Phase. Table 2 shows the results from the post-phase survey and video coding.

Table 2. Post Survey and Video Coding Results for Conduct Phase

\begin{tabular}{lcc}
\hline & $\begin{array}{c}\text { In-Sequence } \\
\mathrm{n}=5\end{array}$ & $\begin{array}{c}\text { Out-of-Sequence } \\
\mathrm{n}=2\end{array}$ \\
\hline Level of Difficulty & & 8 \\
$\quad$ Total & 9 & 1.6 \\
Average & 2.25 & 0.55 \\
SD & 0.5 & \\
Feel Like Engineer & & 24 \\
Total & 11 & 4.8 \\
Average & 2.75 & 0.45 \\
SD & 0.96 & \\
Student Questions & & 36 \\
Total & 12 & 7.2 \\
Average & 2.4 & 1.1 \\
SD & 2.19 & \\
TA Questions & & 23 \\
Total & 12 & 4.6 \\
Average & 2.4 & 2.19 \\
SD & 0.55 &
\end{tabular}


Total $14 \quad 18$

$\begin{array}{lll}\text { Average } & 2.8 & 3.6\end{array}$

SD $1.64 \quad 0.55$

Note. All data collected up to 1:11:04 instead of full three hours due to Swivl video errors.

Both groups perceived the Conduct phase as being less difficult than the Design phase. However, unlike in the Design phase, the out-of-sequence students rated the Conduct phase as easier and they felt more positively about their identity as an engineer than their in-sequence peers.

While out-of-sequence participants perceived the Conduct phase as less difficult, they struggled to complete laboratory tasks without TA assistance. For just the first hour of Conduct phase, the out-of-sequence students had three times as many questions in the first hour than their in-sequence peers. As a result, there were approximately twice as many TA questions directed towards the out-of-sequence participants, ensuring that students were making progress towards task completion.

In spite of the 3:1 ratio of participant questions between the out-of-sequence to the insequence group, there was not a large difference between issues. However, the types of issues between the two groups varied. Most of the issues for the in-sequence students were related to methods rather than concept understanding (e.g., not knowing how to use the temperature probe correctly and issues using Excel). The out-of-sequence group had a wider range of issues (e.g., standard deviation calculations, temperature collection, not understanding how the constraints impact their data, and not knowing how to interpret their graphs to obtain the relevant information about the materials). 
Analyze Phase. Table 3 shows the results from the post-phase survey and video coding protocol.

Table 3. Post Survey and Video Coding Results for Analyze Phase

\begin{tabular}{lcc}
\hline & $\begin{array}{c}\text { In-Sequence } \\
\mathrm{n}=5\end{array}$ & $\begin{array}{c}\text { Out-of-Sequence } \\
\mathrm{n}=5\end{array}$ \\
\hline Level of Difficulty & & 9 \\
$\quad$ Total & 11 & 1.8 \\
Average & 2.2 & 0.45 \\
SD & 0.84 & \\
Feel Like Engineer & & 21 \\
$\quad$ Total & 21 & 4.2 \\
Average & 4.2 & 0.84 \\
SD & 0.84 & \\
Student Questions & & 52 \\
Total & 23 & 10.4 \\
Average & 4.6 & 3.29 \\
SD & 0.55 & \\
TA Questions & & 15 \\
Total & 2 & 3 \\
Average & 0.4 & 0 \\
SD & 0.55 & \\
Issues & & 42 \\
Total & 26 & 8.4 \\
Average & 5.2 & 0.55 \\
SD & 1.64 & \\
\hline
\end{tabular}

Similar to the Conduct phase, the out-of-sequence students rated the Analyze phase as easier than their in-sequence peers. In addition, both groups equally perceived themselves as acting like engineers within the laboratory.

We identified the same pattern regarding the number of questions asked by the students. The out-of-sequence participants asked more than twice the amount of questions than their insequence peers and there were more TA questions directed to this group. There was a large difference in the number of issues between the two semesters. The out-of-sequence participants had nearly twice the number of total issues compared to the in-sequence participants, and there was a variety of reasons for these issues. Out-of-sequence participants struggled with both procedural issues and conceptual issues (e.g., calculations of standard deviation and slope). Furthermore, the out-of-sequence students were unable to meet the final deliverable requirements due to errors in their results which are reflective of the types of issues mentioned. 


\section{Discussion and Implications}

Although perceived and classified as being different, the out-of-sequence students perceived the DC as equally difficult (Design) or even easier (Conduct and Analyze) compared to their insequence peers. This is consistent and related to our previous findings regarding the self-efficacy beliefs of this group of students. The similarity in views regarding task difficulty between the groups could be related to them having similar beliefs regarding their capabilities of performing the course of actions to complete specific tasks. The out-of-sequence students are therefore perceiving the DC as something they could complete on a level equal to the in-sequence group. Similarly, the out-of-sequence participants felt that the DC was engaging and relevant to their engineering major. Students from both groups identified as being an engineer.

During the Design phase, the out-of-sequence students raised more questions and more TA assistance was provided, which could suggest that this group did not have as much background knowledge or previous experience regarding the engineering context or the chemistry concepts. Despite this difference in the frequency of questions asked, both groups had an equal rating of DC difficulty and feelings of being like an engineer, implying that lack of previous understanding of concepts is not discouraging either group in terms of development of their engineering identity.

The data from the Conduct phase provides further insight into differences in participants' understanding of chemistry concepts and laboratory techniques. The out-of-sequence participants had twice the number of questions than the in-sequence participants, the TA asked them more questions, and they had more issues during this phase. Due to the increase in experimental tasks in the Conduct phase, a lot of assistance was sought and help provided for task completion. However, the out-of-sequence participants had very high feelings of being like an engineer and low ratings of difficulty, implying the participants' need for discussions with the TA to move forward with laboratory tasks does not necessarily negatively impact their views of themselves within their major.

There was a very large discrepancy between the two groups during the Analyze phase, which is centered around conceptual understanding and reasoning through a CER and creation of an engineering authentic final deliverable (i.e. technical memo). The high number of issues from out-of-sequence participants during Analyze revealed that the out-of-sequence participants struggled with understanding the primary chemistry concepts in the experiment concerning heat 
capacity, constraints, and data analysis calculations. Regardless of the discrepancy in issues and helps, the feelings of being like an engineer was equal between groups. This again disproves the idea that more issues relates to participants not feeling as successful as engineers, as these participants were able to overcome their issues to complete laboratory tasks. In real engineering contexts, testing and debugging is a key concept to improving procedures, so participants not being discouraged by productive struggle is a good sign for the DC structure.

The relationship between the higher number of questions and issues from the out-of-sequence participants compared to their high rating of feeling like an engineer and low ratings of difficulty reveals that even though the out-of-sequence participants may have appeared to struggle more in the DC, they are still motivated to continue working in the laboratory and do not feel overwhelmed or discouraged by the material or procedures. The data suggests that having a TA available to respond to questions and follow up with the participants is important to ensure outof-sequence participants are supported and can continue making progress.

No significant changes in the structure of DC2 are required to ensure student success for all students, but small clarifications and examples could be added at the beginning of phase periods to reduce issues throughout the DC. Common issues identified for all groups were: calculation errors, which can be reduced by having the TA do a sample calculation at the beginning of class to reduce math errors, which are not related to misunderstanding of the concepts. Furthermore, many issues seen in Conduct and Analyze related to making a graph of the data in Excel and reading the results, and a sample graph construction could be put in the laboratory procedure to streamline this process and reduce repetitive questions about graph construction. Constraints were mentioned by participants as the most confusing part of the laboratory document during the first week of the DC, a review of what the constraints mean during the Analyze phase would likely reduce participant confusion when trying to write the CER tech memo.

The major difference between the in-sequence and out-of-sequence participants observed in this study was a higher rate of questioning and issues from the out-of-sequence participants. However, in spite of these issues, the participants rated their feeling of being like an engineer positively and rated difficulty as fairly easy for each phase. Therefore, as long as out-of-sequence students are receiving assistance throughout the laboratory period, they are able to stay engaged with the materials and are not likely to be discouraged when they encounter issues. TAs should be instructed to be attentive of student needs and provide examples of calculations and material 
construction in order to reduce common errors for all students. The TA should also be aware that out-of-sequence students may need additional assistance understanding engineering and chemistry concepts and to act as facilitators for student learning. If out-of-sequence students are supported throughout the course, they are likely to feel motivated to continue as engineers and continue their trajectory towards completing their engineering major past the introductory chemistry courses.

\section{Acknowledgements}

I would like to thank the University Scholars Program for providing funding to allow me to work with the College of Education and continue my passion for educational research. I would also like to thank the entire ChANgE Chem research team for their undying support of my project and research goals. Lorelie, Corey, and Dr. Crippen have given me a new home in the College of Education, and I am so grateful for their time and mentoring over these past two years. This material is based upon work supported by the National Science Foundation under Grant No. (1625378). Opinions, findings, and conclusions or recommendations expressed in this material are those of the author(s) and do not necessarily reflect the views of the National Science Foundation. The phrase "NAE Grand Challenges for Engineering "TM" and the three-piece puzzle logos are trademarks of the National Academy of Sciences on behalf of the National Academy of Engineer. Use of the trademarks and NAE Grand Challenges for Engineering graphic icons are used with permission of the National Academy of Sciences/National Academy of Engineering.

\section{References}

Hofstein, A., \& Lunetta, V. N. (2004). The laboratory in science education: Foundations for the twentyfirst century. Science Education, 88(1), 28-54. doi:10.1002/sce.10106

Johri, A., \& Olds, B. M. (2011). Situated engineering learning: Bridging engineering education research and the learning sciences. Journal of Engineering Education, 100(1), 151-185. doi:10.1002/j.2168-9830.2011.tb00007.x

Krapp, A., \& Prenzel, M. (2011). Research on Interest in Science: Theories, methods, and findings. International Journal of Science Education, 33(1), 27-50. doi:10.1080/09500693.2010.518645

National Academy of Engineering. (n.d.). NAE grand challenges for engineering. Retrieved February 10, 2020, from http://www.engineeringchallenges.org/

Ohland, M. W., Sheppard, S. D., Lichtenstein, G., Eris, O., Chachra, D., \& Layton, R. A. (2008). Persistence, Engagement, and Migration in Engineering Programs. Journal of Engineering Education, 97(3), 259-278. doi:10.1002/j.2168-9830.2008.tb00978.x

Payne, C., \& Crippen, K. J., \& Imperial, L., \& Wu, C., \& Brucat, P. J., \& Korolev, M. (2019, June), Board 33: Persistence of First-year Engineering Majors with a Design-based Chemistry Laboratory Curriculum In- and Out-of-Sequence Paper presented at 2019 ASEE Annual Conference \& Exposition, Tampa, Florida. https://peer.asee.org/32326

Popper, K. (1999). All life is problem solving. London: Routledge.doi:10.1080/09500693.2010.518645 


\section{Appendix A}

Video Coding Protocol

\begin{tabular}{|l|l|l|}
\hline Code & Definition & When to Use \\
\hline Issue & $\begin{array}{l}\text { scientific, } \\
\text { mathematical, } \\
\text { engineering } \\
\text { concept; } \\
\text { the prescribed set } \\
\text { of steps or protocol } \\
\text { for a method }\end{array}$ & $\begin{array}{l}\text { when participants missed one or more steps or } \\
\text { inappropriately conducted steps of a method } \\
\text { or skill, or performed unnecessary steps AND } \\
\text { such behavior leads or potentially leads to } \\
\text { frustration or task failure; } \\
\text { when participants perform any inaccurate or } \\
\text { naïve action or statement about the chemistry } \\
\text { concept or engineering practice }\end{array}$ \\
\hline Student Questions & Questions prompted by the students to aid in task completion \\
\hline TA Questions & $\begin{array}{l}\text { Help, advice or suggestion prompted by the TAs (but not sought by } \\
\text { the students) to aid in task completion }\end{array}$ \\
\hline
\end{tabular}

